\title{
WHY IS VISION IMPAIRED IN FRAGILE X PREMUTATION CARRIERS? THE ROLE OF FRAGILE X MENTAL RETARDATION PROTEIN AND POTENTIAL FMR1 MRNA TOXICITY
}

\author{
S. KÉRI ${ }^{a, b *}$ AND G. BENEDEK ${ }^{a}$ \\ a University of Szeged, Department of Physiology, Szeged, Hungary \\ ${ }^{b}$ National Psychiatry Center, Budapest, Hungary
}

\begin{abstract}
Dysfunctions of the geniculo-striatal magnocellular (M) visual pathway and its cortical recipients have been documented in fragile $X$ syndrome and in FMR1 premutation carriers. However, the mechanism of this impairment is less clear. To elucidate this issue, we completed the measurement of visual functions at different stages of information processing: low-level mechanisms (contrast sensitivity biasing information processing toward the $M$ and parvocellular $[P]$ pathways), primary visual cortex (motion-defined and static Vernier threshold), and higher-level form and motion processing (coherence thresholds). Results revealed that FMR1 premutation carriers, relative to non-carrier controls, exhibited lower contrast sensitivity for M pathway-biased stimuli, higher Vernier threshold for motion-defined stimuli, and higher global motion coherence threshold. Although both elevated FMR1 mRNA and reduced fragile $X$ mental retardation protein (FMRP) levels were associated with impaired visual functions, regression analysis indicated that FMRP was the primary factor. In premutation carriers, a toxic gainof-function of elevated FMR1 mRNA has been suggested, whereas reduced FMRP is linked to neurodevelopmental aspects. Here, we showed that FMRP may the primary factor associated with visual dysfunctions. () 2012 IBRO. Published by Elsevier Ltd. All rights reserved.
\end{abstract}

Key words: fragile $X$ syndrome, premutation carrier, neurodevelopment, vision, RNA toxicity.

Fragile $\mathrm{X}$ syndrome (FXS) is a prevalent form of inherited neurodevelopmental disorders leading to intellectual disability. In the full syndrome, the fragile $X$ mental retardation protein (FMRP) is absent, which is a consequence of FMR1 gene silencing. The mechanism of gene silencing is based on the expansion of a CGG trinucleotide repeat (Xq27.3, >200 repeats in the full syndrome) and an increased methylation of the promoter region (O'Donnell and Warren, 2002; Bear et al., 2008; Walter et al., 2009; Rousseau et al., 2011). FMRP is a widespread negative regulator of translation, and therefore its absence leads to the

${ }^{*}$ Correspondence to: S. Kéri, University of Szeged, Department of Physiology, Dóm sq. 10, Szeged, Hungary. Tel: +36-20-448-3530; fax: +36-62-545-842

E-mail address: szkeri2000@yahoo.com or keri.szabolcs.gyula@ med.u-szeged.hu (S. Kéri).

Abbreviations: FMRP, fragile $X$ mental retardation protein; FXS, fragile $X$ syndrome; $M$, magnocellular; mRNA, messenger RNA; $P$, parvocellular; Tukey HSD, Tukey honestly significant difference. increased translation of several genes (De Rubeis and Bagni, 2011).

In contrast to full FXS, in FMR1 premutation carriers the size of the CGG expansion is between 55 and 200 repeats, which is associated with a subtle cognitive and neuropsychiatric phenotype (Hagerman et al., 1996; Bennetto et al., 2001; Steyaert et al., 2003; Moorem et al., 2004; Cornish et al., 2005, 2009; Bourgeois et al., 2009; Boyle and Kaufmann, 2010; but see Franke et al., 1999). In premutation carriers, there is no absolute FMR1 gene silencing. The expression of expanded CGG triplets in messenger RNA (mRNA) may have negative consequences, resulting in premature ovarian insufficiency and fragile X-associated tremor/ataxia syndrome (Hagerman and Hagerman, 2004; Berman and Wilemsen, 2009; Hunter et al., 2010). This toxic gain-of-function of excess mRNA is complicated by the fact that there is an association among reduced FMRP, increased FMR1 transcription, and CGG repeat number in intermediate-length and premutation carriers (Kenneson et al., 2001), which is because of a less efficient initiation of translation (Ludwig et al., 2011). This may also contribute to dysfunctions observed in premutation carriers (Hessl et al., 2011). Lower FMRP expression may be associated with various psychiatric disorders, including schizophrenia, depression, and anxiety (Fatemi and Folsom, 2011; Qin et al., 2011).

Our aim was to study how increased FMR1 mRNA and decreased FMRP levels contribute to visual dysfunction in premutation carriers. Patients with FXS and premutation carriers display visual perceptual anomalies, which are characterized by the impairment of the precortical magnocellular (M) pathway and its cortical targets (Kogan et al., 2004a,b; Farzin et al., 2008; Kéri and Benedek, 2009, 2010). Cells of the M pathway can be stimulated by low luminance contrast, low spatial frequencies (coarse resolution of objects), and rapid temporal changes. In contrast, parvocellular $(P)$ pathways prefer static patterns with medium and high spatial frequency (fine details of objects) and colors (Van Essen and Gallant, 1994; Nassi and Callaway, 2009). After an interaction in the primary visual cortex (V1), M pathways give an intensive afferentation to cortical areas responsible for motion perception, detection of spatial location, and visuomotor coordination (dorsal occipitoparietal stream). $\mathrm{P}$ pathways give afferents to ventral occipitotemporal regions responsible for color perception and object recognition (Van Essen and Gallant, 1994; Nassi and Callaway, 2009).

Kogan et al. (2004a) and Zangenehpour et al. (2009) demonstrated that the $\mathrm{M}$ layers of the lateral geniculate 
Table 1. Demographic and molecular characteristics

\begin{tabular}{lcc}
\hline & $\begin{array}{l}\text { FMR1 premutation carriers } \\
(n=21)\end{array}$ & $\begin{array}{l}\text { Controls } \\
(n=20)\end{array}$ \\
\hline Age (y) & $32.3(8.6)$ & $34.9(10.5)$ \\
Education (y) & $12.6(3.9)$ & $12.4(4.4)$ \\
IQ & $105.9(11.3)$ & $104.8(10.5)$ \\
CGG* $^{*}$ & $110.4(30.0)$ & $25.3(3.4)$ \\
FMR1* $^{*}$ & $3.6(1.7)$ & $1.3(0.2)$ \\
FMRP $^{*}$ & $68.2(40.4)$ & $134.2(79.4)$ \\
\hline
\end{tabular}

Data are mean (standard deviation).

${ }^{*} P<0.001$

nucleus exhibit high FMRP expression, which suggests that these neurons are especially vulnerable in FXS. In accordance with these findings, we found a positive relationship between FMRP expression in lymphocytes and M pathway/dorsal stream functions in healthy volunteers (Kéri and Benedek, 2011). The fact that FXS patients present visual impairments indicates that visual dysfunctions in premutation carriers may be because of the reduced FMRP levels, rather than the increased FMR1 mRNA expression. In the present study, we directly investigated how FMR1 mRNA levels, a potential marker of neuronal toxicity in premutation carriers, and FMRP expression contribute to visual functions.

\section{EXPERIMENTAL PROCEDURES}

\section{Participants}

We enrolled 21 men with FMR1 premutation (sons of female premutation carriers) and 20 control volunteers (Table 1). All participants underwent molecular biological assessment (see Molecular biological measurements) and detailed neurological and psychiatric examination. None of the participants exhibited signs and symptoms of neurological or mental disorders. General intellectual functions were evaluated with the Wechsler Adult Intelligence Scale-III (Wechsler, 1997). Exclusion criteria included renal, liver, cardiac, and endocrinological diseases, history of head trauma, migraine, and alcohol or drug abuse. All participants gave written informed consent, and the institutional ethics board approved the study.

\section{Visual contrast sensitivity}

We used the method described in our previous studies (Kéri and Benedek, 2009, 2011). Stimuli were vertical sinusoidal luminancecontrast gratings. The gratings used to bias information processing toward the $\mathrm{M}$ and $\mathrm{P}$ pathways had different spatiotemporal properties (M pathway-spatial frequency: 0.3 cycle/degree, temporal frequency: $10 \mathrm{~Hz}$; P pathway-spatial frequency: $10 \mathrm{cycles/}$ degree, temporal frequency: $1 \mathrm{~Hz})($ Fig. 1). The stimulus area was circular (diameter: 8 degrees of visual angle, luminance: $31 \mathrm{~cd} / \mathrm{m}^{2}$, initial Michelson contrast: $12 \%$ ). This level was increased or de-

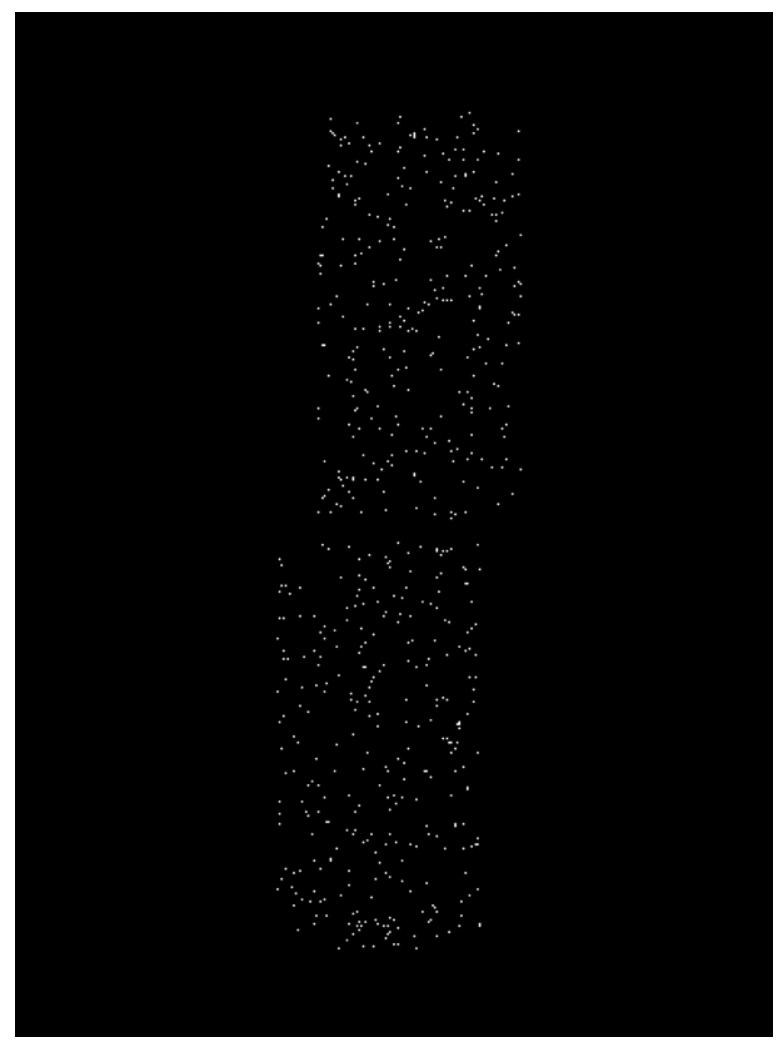

Fig. 2. Vernier stimuli. The vertical bars were composed of white dots against a black background. There was a horizontal displacement between the bars.

creased according to a Yes/No one-up/two-down staircase procedure. Participants entered the responses by pressing one of two keys on the computer keyboard. The staircase was finished when the slope and SD of the last 12 trials were less than the step size. The detection thresholds were the mean value of the last 12 reversals.

\section{Static and motion-defined Vernier}

We adopted the method of McKendrick et al. (2006). Stimuli were two vertical bars, which were defined either by static white dots (luminance: $100 \mathrm{~cd} / \mathrm{m}^{2}$ ) against a black background (luminance: $0.5 \mathrm{~cd} / \mathrm{m}^{2}$ ) or by the relative motion of randomly placed dots (Fig. 2). The vertical bars were characterized by the following parameters: dot size (17 s arc-square pixels), horizontal and vertical extent (10 min arc wide and $15 \mathrm{~min}$ arc high), and vertical offset (4 min arc). In this static condition, the dots did not move on the screen.

In the motion-defined condition, a central circular area (diameter: 1.23 degrees of visual angle) was first covered with randomly placed dots (density: 4593 dots $/{ }^{\circ 2}$ ), and therefore no bar stimuli appeared on the screen. The bars became visible when the background dots moved upward (speed: 1.25 degrees/s), whereas the

\section{Low Contrast}

\section{High Contrast}

Fig. 1. Illustration of gratings used for contrast sensitivity measurements. 


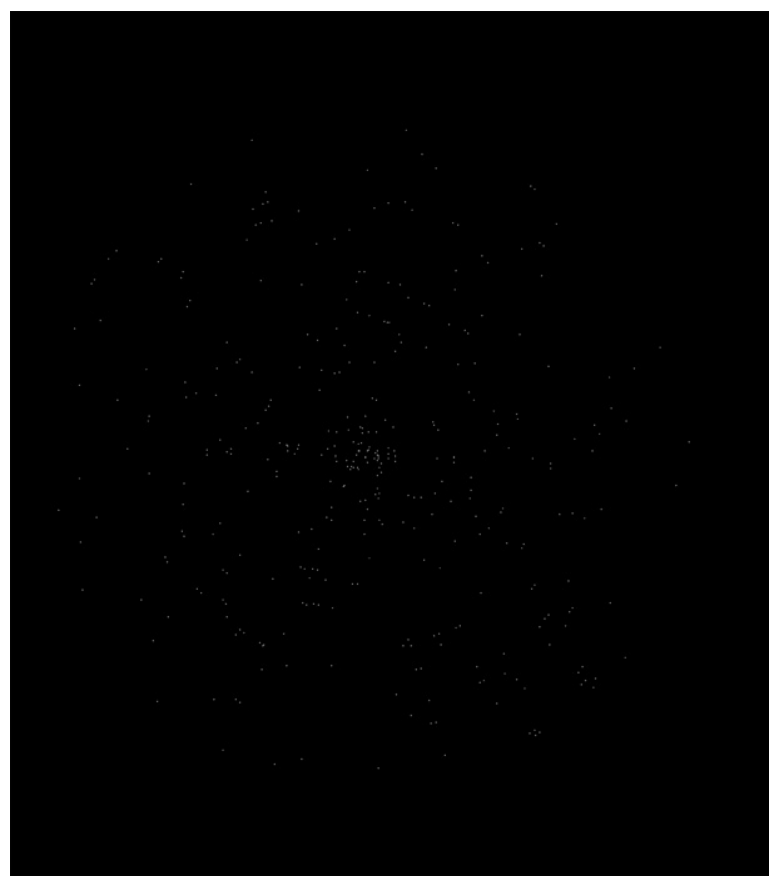

Fig. 3. Illustration of Glass pattern stimuli used for coherence threshold measurements.

dots comprising the bars moved downward at the same speed (eight frame motion sequence, stimulus duration: $456 \mathrm{~ms}$ ). Dot density and stimulus duration were the same in the static and motion-defined conditions.

We measured thresholds with a single-interval, forced-choice procedure. In the test interval, stimuli were presented with a variable degree of horizontal displacement (left or right in a randomized order) between the upper and lower bars (Fig. 2). Participants were asked to decide whether the bars were displaced or not (they were not informed that stimuli are necessarily displaced, and filler trials with no displacement were included). The horizontal offset of the bars was reduced in the case of three consecutive correct responses and was increased after each incorrect response (three-down/one-up staircase converging on $79 \%$ correct performance). Two staircases were interleaved. The initial displacement of the bars was 2 min arc for the static condition and 6 min arc for the motion-defined condition. The initial staircase step size was $20 \mathrm{~s}$ arc, which was halved on the first two reversals. The staircases were completed after six reversals. The result of the staircase was the average of the final four reversals. The mean of the two interleaved thresholds was the final dependent measure.

\section{Form and motion coherence}

For the measurement of coherence thresholds, we used Glass pattern stimuli (Glass, 1969; McKendrick et al., 2006). Glass patterns consisted of dot pairs randomly placed within a circular stimulus area (distance between dots: $9 \mathrm{~min}$ arc, diameter of stimulus area: 10 degrees of visual angle, number of dots: 200). The luminance of the dots and background were identical to that used in the Vernier test. The pattern was concentric, which means that dot pairs were perpendicular to the center of the image (Fig. 3 ). During the measurement of form coherence threshold, some coherent dots were replaced with randomly positioned noise dots. For example, in a $50 \%$ coherence pattern, half of the dots were paired and positioned concentrically, whereas half of the dots were placed randomly.

The size and luminance of the dots were the same in the form and motion condition. Motion was generated similarly to the mo- tion-defined Vernier test. A percentage of randomly chosen dots moved in the signal direction, whereas all other dots moved in random directions (9 min arc/frame, 3 degrees/s).

We measured thresholds with a single-interval, forced-choice procedure. Participants indicated whether the interval contained a concentric structure (form coherence task) or downward motion (motion coherence task). As in the case of the Vernier task, we used a three-down/one-up staircase. The initial proportion of signal dots was $50 \%$. The initial staircase step was $4 \%$, which was halved after the first reversal. All other parameters of the staircase measurement were the same as described in the Vernier task.

\section{Molecular biological measurements}

Peripheral blood was drawn from the cubital vein of the participants. CGG repeat size was determined using standard Southern blot analysis as described by Steyaert et al. (2003). FMR1 mRNA was measured using Affymetrix Quantigene (Vala Sciences Inc., CA, USA) based on the protocol of Tassone et al. (2000).

For the quantitative measurement of FMRP levels, we used the new enzyme-linked immunosorbent assay (ELISA) method (Iwahashi et al., 2009). The pelleted lymphocytes were suspended in lysis buffer, MPer (Pierce, Rockford, IL, USA) and protease inhibitor set III (Calbiochem, San Diego, CA, USA). We used bicinchoninic acid protein assay kit (Pierce, Rockford, IL, USA) for the quantification of protein concentration. In order to avoid protein aggregation, we prepared a maltose binding protein (MBP)-FMRP fusion. The peptide sequence (KDRNQKKEKPDSVD), which is located near the carboxy terminus of FMRP, was used for the production of chicken antibody (AVES Labs, Inc., Tigard, OR, USA). Well plates were coated with chicken anti-FMRP antibody, detection antibody, and horseradish peroxidase-conjugated antimouse antibody. Luminescence was read with a Chiron luminometer (Labequip, Markham, Ontario, Canada) (for the details of the protocol, see Iwahashi et al., 2009).

\section{RESULTS}

\section{Visual contrast sensitivity}

Fig. 4 presents the contrast sensitivity results. We conducted a group (premutation carriers vs. controls) by stimulus type (M vs. P pathway) repeated measures analysis of variance (ANOVA), which revealed significant main effects of group $(F(1,39)=16.72, \quad P<0.001, \eta=0.30)$, stimulus type $(F(1,39)=6.90, P<0.05, \eta=0.15)$, and a two-way interaction between them $(F(1,39)=14.34, \quad P<0.005$, $\eta=0.27$ ). Tukey honestly significant difference (Tukey HSD) tests revealed that premutation carriers had lower contrast sensitivity than controls in the $\mathrm{M}$ pathway condition $(P<0.001)$, but not in the $\mathrm{P}$ pathway condition $(P>0.5)$ (Fig. 4).

\section{Vernier threshold}

Fig. 5 presents the Vernier threshold results. A similar ANOVA was conducted on these results as we used for the analysis of contrast sensitivity, but here stimulus type was static vs. motion-defined Vernier. There were significant main effects of group $(F(1,39)=7.90, P<0.05, \eta=0.16)$, stimulus type $(F(1,39)=14.56, P<0.001 \mathrm{~m}, \eta=0.27)$, and a two-way interaction between them $(F(1,39)=6.72$, $P<0.05, \eta=0.15)$. Tukey HSD tests revealed that premutation carriers displayed higher Vernier thresholds than controls only in the motion-defined condition $(P<0.05$; static: $P>0.5$ ). 


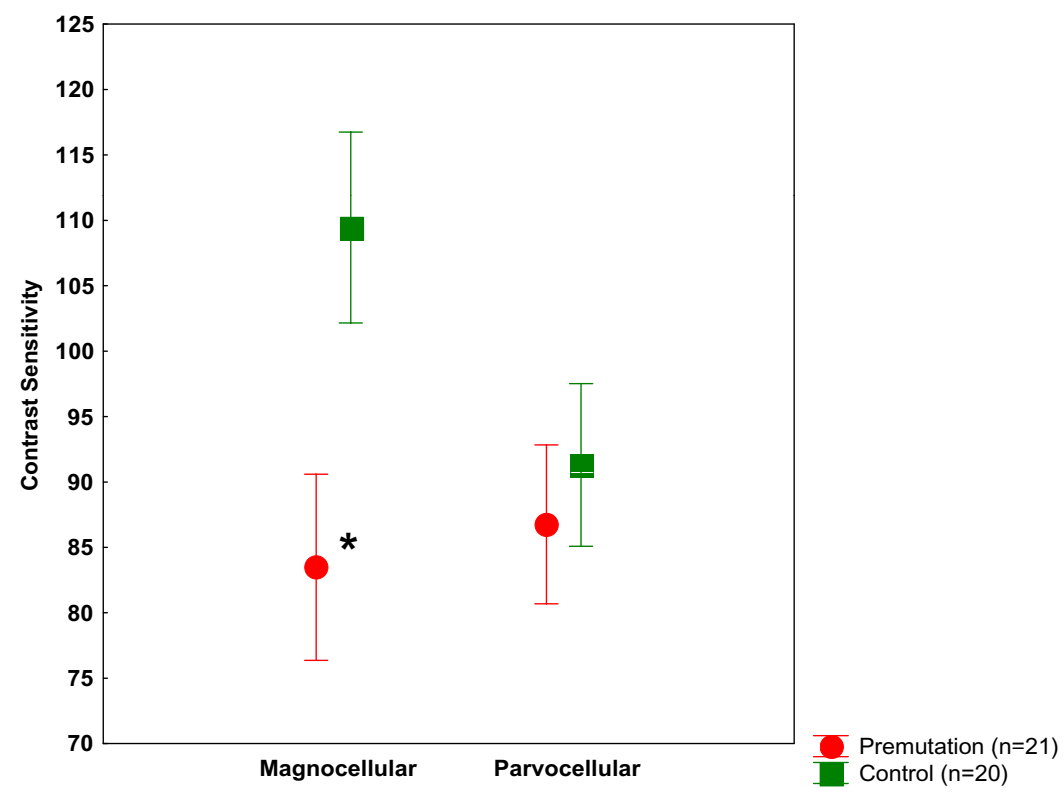

Fig. 4. Visual contrast sensitivity results. Error bars indicate $95 \%$ confidence intervals. ${ }^{*} P<0.001$ (comparison of $F M R 1$ premutation carriers and controls, Tukey HSD tests).

\section{Form and motion coherence threshold}

Fig. 6 depicts the coherence threshold values. As in the Vernier test, there were significant main effects of group $(F(1,39)=4.18, \quad P<0.05, \quad \eta=0.10), \quad$ stimulus type $(F(1,39)=13.35, P<0.005, \eta=0.25)$, and a two-way interaction between them $(F(1,39)=7.27, \quad P<0.05$, $\eta=0.16)$. Premutation carriers had elevated coherence threshold only in the motion condition relative to controls (Tukey HSD, $P<0.05$; form condition: $P>0.5$ ).

\section{Molecular measures and visual psychophysics}

FMRP and FMR1 mRNA levels are depicted in Table 1. In the premutation group, there was a significant positive correlation between CGG size and FMR1 mRNA level (Spearman's $R=0.82, P<0.001$ ), and a tendency between FMR1 mRNA and FMRP levels $(R=-0.38, P=0.09)$. Table 2 shows the correlations among FMR1 mRNA/FMRP levels and visual test measures. In general, both FMR1 mRNA and FMRP levels correlated with data from M pathway-biased and motion-related tests: higher FMR1 mRNA

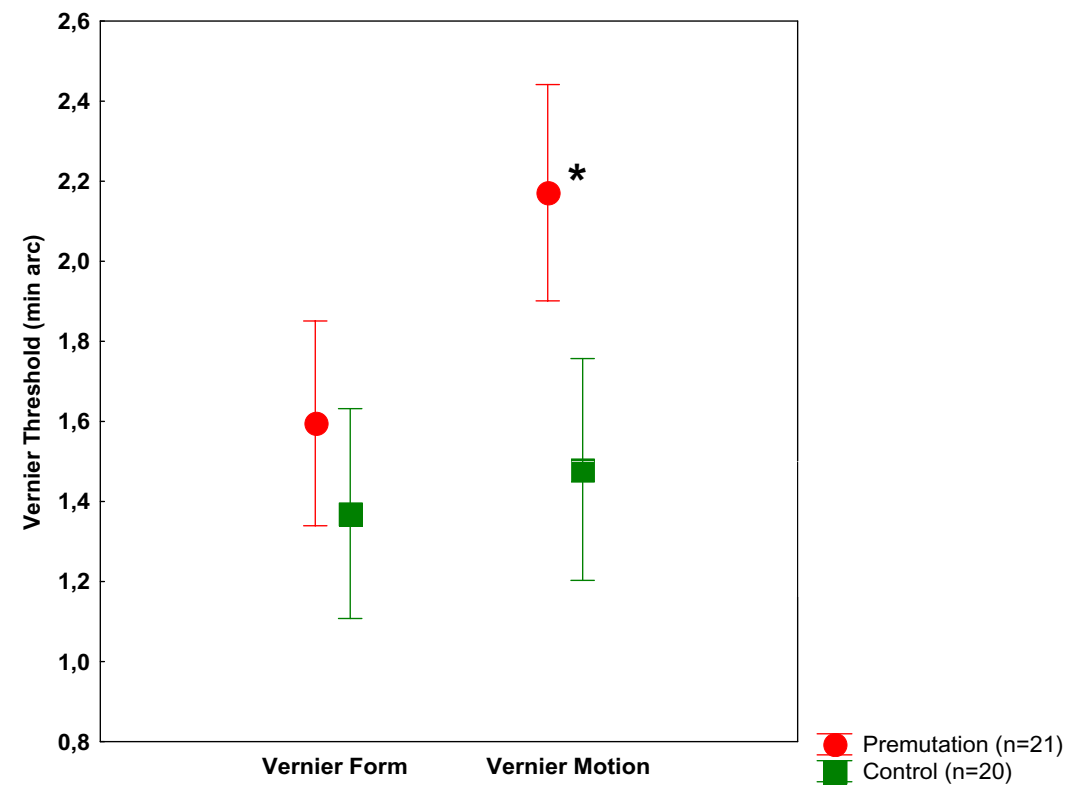

Fig. 5. Vernier threshold results. Error bars indicate $95 \%$ confidence intervals. ${ }^{*} P<0.05$ (comparison of $F M R 1$ premutation carriers and controls, Tukey HSD tests). 


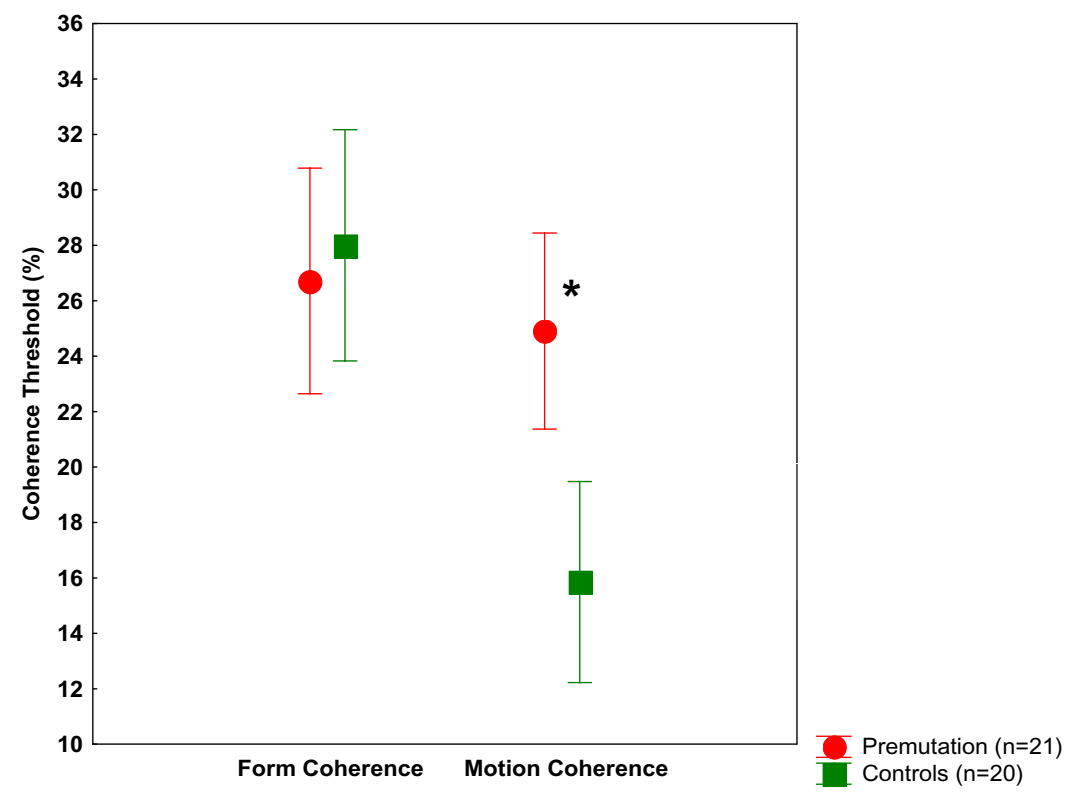

Fig. 6. Global form and motion coherence thresholds. Error bars indicate $95 \%$ confidence intervals. ${ }^{*} P<0.05$ (comparison of $F M R 1$ premutation carriers and controls, Tukey HSD tests).

levels and lower FMRP levels were associated with less efficient visual processing (Table 2).

When a multiple regression analysis was conducted to determine the predictors of $\mathrm{M}$ pathway-related contrast sensitivity, FMRP level was significant $\left(b^{*}=0.51\right.$, $t(18)=2.55, P<0.05)$, whereas FMR1 mRNA level did not retain significance $(P>0.1)$. In the case of motion-defined Vernier, neither FMRP nor FMR1 mRNA reached the level of significance $(P>0.1)$, whereas in the case of motion coherence, again, only FMRP was significant $\left(b^{*}=-0.48\right.$, $t(18)=-2.44, P<0.05)$. In accordance with correlation analyses, regression analyses did not reveal significant predictors for $\mathrm{P}$ pathway-biased contrast sensitivity, static Vernier, and form coherence threshold $(P>0.1)$.

\section{DISCUSSION}

In line with results from previous studies (Kéri and Benedek, 2009, 2010), we found a selective impairment of visual perceptual functions in FMR1 premutation carriers. Specifically, contrast sensitivity biasing information processing toward the M pathways (precortical mechanisms), motion-defined Vernier, which assesses the functioning of the primary visual cortex (V1), and motion coherence were impaired, whereas all tests related to the $P$ pathways and its cortical recipients were spared.
Although visual contrast sensitivity dysfunctions for gratings with low-spatial and high-temporal frequency were directly related to the pathology of $M$ cells in the lateral geniculate nucleus of patients with FXS (Kogan et al., 2004a), evidence for Vernier stimuli is indirect. It has been shown, however, that early cortical integration is critical for Vernier performance (Barlow, 1981; Wilson, 1986; Victor and Conte, 2000; Duncan and Boynton, 2003). The static and motion-defined stimuli used in this study may distinctly investigate the spatial localization capacity of form and motion pathways (Regan, 1986; Banton and Levi, 1993; McKendrick et al., 2006). In our previous study, FMR1 premutation carriers displayed elevated Vernier thresholds only for stimuli biasing information processing toward the $\mathrm{M}$ pathways (Kéri and Benedek, 2009).

According to the concept of the hierarchical organization of the visual system, local stimulus attributes processed in V1 are integrated in the extrastriate cortex, including the middle temporal (MT)/V5/V3a and lateral occipital complex (LOC)/V4 assembling local information into global motion and form representations, respectively (Tootell et al., 2003; Orban et al., 2004). At this integrative stage of information processing, FMR1 premutation carriers again showed a selective deficit for motion stimuli,

Table 2. Correlations between molecular measures and visual functions

\begin{tabular}{lccrrrr}
\hline & Contrast magnocellular & Contrast parvocellular & Vernier form & Vernier motion & Form coherence & Motion coherence \\
\hline FMR1 & -0.38 & -0.1 & 0.13 & $0.45^{*}$ & -0.02 \\
FMRP & $0.58^{*}$ & 0.2 & -0.01 & $-0.48^{*}$ & 0.06 & $-0.57^{*}$ \\
\hline
\end{tabular}

The table shows Spearman's correlation coefficients (Rs).

FMR1, fragile $X$ mental retardation mRNA; FMRP, fragile $X$ mental retardation protein.

${ }^{*} P<0.05$. 
which is consistent with previous observations (Kéri and Benedek, 2010).

The critical question of the study was whether these dysfunctions are related to decreased FMRP or increased FMR1 mRNA levels, a putative toxic gain-offunction mechanism of increased expression of extended CGG repeats (Hagerman et al., 2010). Although correlation analysis revealed that both decreased FMRP and increased FMR1 mRNA levels were associated with less efficient visual processing, regression analyses indicated that FMRP was the primary factor. This is highly reminiscent to that found in the case of amygdala functions in FMR1 permutation carriers, which correlated with both FMRP and FMR1 mRNA, but FMRP was the primary factor (Hessl et al., 2011). A confirmatory aspect of our study is that, by using a more sensitive method than the assessment of the number of FMRP positive lymphocytes, we replicated the relationship between visual functions and FMRP expression (Kéri and Benedek, 2011).

Beyond the measurement of FMRP and FMR1 mRNA levels, there are major differences between our previous work (Kéri and Benedek, 2009, 2010) and the present one. First, in this study we investigated male premutation carriers, which is a much straightforward approach because of the presence of a single $\mathrm{X}$ chromosome in these individuals (female carriers have a functional copy of the gene that is expressed randomly in $50 \%$ of cells). Moreover, from a clinical point of view, male premutation carriers are at a higher risk of RNA toxicity than female premutation carriers (Hagerman et al., 2010), which warrants the investigation of potential toxicity markers (e.g. visual dysfunctions). Finally, we used a psychometrically improved stimulus set, that is, motion- and form-defined Glass patterns, which allow a more exact investigation of visual information processing.

The results of the present study may have an impact on the differentiation of neurodevelopmental vs. neurodegenerative aspects of fragile $X$ premutation-related diseases. Increased FMR1 mRNA is a risk factor for late-onset neurodegenerative processes in premutation carriers, leading to fragile X-associated tremor/ataxia syndrome. In contrast, decreased FMRP may represent the neurodevelopmental aspect of the disease (Hagerman and Hagerman, 2004; Rousseau et al., 2011). Future longitudinal studies should clarify whether visual dysfunctions are associated with neurodevelopmental changes or with neurodegeneration, although the primacy of FMRP over FMR1 mRNA supports the neurodevelopmental hypothesis.

The present study has several limitations. First, behavioral measures were correlated with peripheral markers from the blood, and no functional brain imaging was used during the tasks. Nevertheless, the consistent and uniform pattern of results across different tests is against the likelihood of chance findings. FMRP expression has been documented in the $\mathrm{M}$ layers of the lateral geniculate nucleus (Kogan et al., 2004a; Zangenehpour et al., 2009), which is an indirect support for our findings. Second, although statistical effect size values reflected adequate power, the sample size was relatively small. Therefore, future studies must confirm the results in an independent group of FMR1 premutation carriers.

Acknowledgments-We are indebted to András Kovács, Zsolt Balog, and Zsuzsanna Halmai for technical assistance. The study was supported by the Hungarian Research Fund (OTKA NF72488, K83810).

\section{REFERENCES}

Banton T, Levi DM (1993) Spatial localization of motion-defined and luminance-defined contours. Vision Res 33:2225-2237.

Barlow HB (1981) Critical limiting factors in the design of the eye and the visual cortex. Proc R Soc Lond B Biol Sci 212:1-34.

Bear MF, Dölen G, Osterweil E, Nagaraja N (2008) Fragile X: translation in action. Neuropsychopharmacology 33:84-87.

Bennetto L, Pennington BF, Porter D, Taylor AK, Hagerman RJ (2001) Profile of cognitive functioning in women with the fragile $X$ mutation. Neuropsychology 15:290-299.

Berman RF, Willemsen R (2009) Mouse models of fragile X-associated tremor ataxia. J Investig Med 57:837-841.

Bourgeois JA, Coffey SM, Rivera SM, HessI D, Gane LW, Tassone F, Greco C, Finucane B, Nelson L, Berry-Kravis E, Grigsby J, Hagerman PJ, Hagerman RJ (2009) A review of fragile $X$ premutation disorders: expanding the psychiatric perspective. J Clin Psychiatry 70:852-862.

Boyle L, Kaufmann WE (2010) The behavioral phenotype of FMR1 mutations. Am J Med Genet C Semin Med Genet 154C:469-476.

Cornish K, Kogan C, Turk J, Manly T, James N, Mills A, Dalton A (2005) The emerging fragile $X$ premutation phenotype: evidence from the domain of social cognition. Brain Cogn 57:53-60.

Cornish KM, Kogan CS, Li L, Turk J, Jacquemont S, Hagerman RJ (2009) Lifespan changes in working memory in fragile $X$ premutation males. Brain Cogn 69:551-558.

De Rubeis S, Bagni C (2011) Regulation of molecular pathways in the fragile $X$ syndrome: insights into autism spectrum disorders. J Neurodev Disord 3:257-269.

Duncan RO, Boynton GM (2003) Cortical magnification within human primary visual cortex correlates with acuity thresholds. Neuron 38:659-671.

Farzin F, Whitney D, Hagerman RJ, Rivera SM (2008) Contrast detection in infants with fragile $X$ syndrome. Vision Res 48:1471-1478.

Fatemi SH, Folsom TD (2011) The role of fragile $X$ mental retardation protein in major mental disorders. Neuropharmacology 60:1221-1226.

Franke P, Leboyer M, Hardt J, Sohne E, Weiffenbach O, Biancalana VV, Cornillet-Lefebre P, Delobel B, Froster U, Schwab SG, Poustka F, Hautzinger M, Maier W (1999) Neuropsychological profiles of FMR-1 premutation and full-mutation carrier females. Psychiatry Res 87:223-231.

Glass L (1969) The Moire effect from random dots. Nature 223:578-580.

Hagerman PJ, Hagerman RJ (2004) Fragile X-associated tremor/ ataxia syndrome (FXTAS). Ment Retard Dev Disabil Res Rev 10:25-30.

Hagerman R, Hoem G, Hagerman P (2010) Fragile X and autism: intertwined at the molecular level leading to targeted treatments. Mol Autism 1:12.

Hagerman RJ, Staley LW, O'Conner R, Lugenbeel K, Nelson D, McLean SD, Taylor A (1996) Learning-disabled males with a fragile $X$ CGG expansion in the upper premutation size range. Pediatrics 97:122-126.

HessI D, Wang JM, Schneider A, Koldewyn K, Le L, Iwahashi C, Cheung K, Tassone F, Hagerman PJ, Rivera SM (2011) Decreased fragile $X$ mental retardation protein expression underlies amygdala dysfunction in carriers of the fragile $X$ premutation. Biol Psychiatry 70:859-865. 
Hunter JE, Rohr JK, Sherman SL (2010) Co-occurring diagnoses among FMR1 premutation allele carriers. Clin Genet 77:374-381.

Iwahashi C, Tassone F, Hagerman RJ, Yasui D, Parrott G, Nguyen D, Mayeur G, Hagerman PJ (2009) A quantitative ELISA assay for the fragile $X$ mental retardation 1 protein. J Mol Diagn 11:281-289.

Kenneson A, Zhang F, Hagedorn CH, Warren ST (2001) Reduced FMRP and increased FMR1 transcription is proportionally associated with CGG repeat number in intermediate-length and premutation carriers. Hum Mol Genet 10:1449-1454.

Kéri S, Benedek G (2009) Visual pathway deficit in female fragile $X$ premutation carriers: a potential endophenotype. Brain Cogn 69:291-295.

Kéri S, Benedek G (2010) The perception of biological and mechanical motion in female fragile $X$ premutation carriers. Brain Cogn 72:197-201

Kéri S, Benedek G (2011) Fragile X protein expression is linked to visual functions in healthy male volunteers. Neuroscience 192:345-350.

Kogan CS, Boutet I, Cornish K, Zangenehpour S, Mullen KT, Holden JJ, Der Kaloustian VM, Andermann E, Chaudhuri A (2004a) Differential impact of the FMR1 gene on visual processing in fragile $X$ syndrome. Brain 127:591-601.

Kogan CS, Bertone A, Cornish K, Boutet I, Der Kaloustian VM, Andermann E, Faubert J, Chaudhuri A (2004b) Integrative cortical dysfunction and pervasive motion perception deficit in fragile $X$ syndrome. Neurology 63:1634-1639.

Ludwig AL, Hershey JW, Hagerman PJ (2011) Initiation of translation of the FMR1 mRNA occurs predominantly through 5'-end-dependent ribosomal scanning. J Mol Biol 407:21-34.

McKendrick AM, Badcock DR, Gurgone M (2006) Vernier acuity is normal in migraine, whereas global form and global motion perception are not. Invest Ophthalmol Vis Sci 47:3213-3219.

Moorem CJ, Daly EM, Schmitz N, Tassone F, Tysoe C, Hagerman RJ, Hagerman PJ, Morris RG, Murphy KC, Murphy DG (2004) A neuropsychological investigation of male premutation carriers of fragile X syndrome. Neuropsychologia 42:1934-1947.

Nassi JJ, Callaway EM (2009) Parallel processing strategies of the primate visual system. Nat Rev Neurosci 10:360-372.

O'Donnell WT, Warren ST (2002) A decade of molecular studies of fragile X syndrome. Annu Rev Neurosci 25:315-338.
Orban GA, Van Essen D, Vanduffel W (2004) Comparative mapping of higher visual areas in monkeys and humans. Trends Cogn Sci 8:315-324.

Qin M, Xia Z, Huang T, Smith CB (2011) Effects of chronic immobilization stress on anxiety-like behavior and basolateral amygdala morphology in Fmr1 knockout mice. Neuroscience 194:282-290.

Regan D (1986) Form from motion parallax and form from luminance contrast: vernier discrimination. Spat Vis 1:305-318.

Rousseau F, Labelle Y, Bussières J, Lindsay C (2011) The fragile $X$ mental retardation syndrome 20 years after the FMR1 gene discovery: an expanding universe of knowledge. Clin Biochem Rev 32:135-162.

Steyaert J, Legius E, Borghgraef M, Fryns JP (2003) A distinct neurocognitive phenotype in female fragile- $X$ premutation carriers assessed with visual attention tasks. Am J Med Genet A 116:44-51.

Tassone F, Hagerman RJ, Taylor AK, Gane LW, Godfrey TE, Hagerman PJ (2000) Elevated levels of FMR1 mRNA in carrier males: a new mechanism of involvement in the fragile-X syndrome. Am J Hum Genet 66:6-15.

Tootell RB, Tsao D, Vanduffel W (2003) Neuroimaging weighs in: humans meet macaques in "primate" visual cortex. J Neurosci 23:3981-3989.

Van Essen DC, Gallant JL (1994) Neural mechanisms of form and motion processing in the primate visual system. Neuron 13:1-10.

Victor JD, Conte MM (2000) Two-frequency analysis of interactions elicited by Vernier stimuli. Vis Neurosci 17:959-973.

Walter E, Mazaika PK, Reiss AL (2009) Insights into brain development from neurogenetic syndromes: evidence from fragile $X$ syndrome, Williams syndrome, Turner syndrome and velocardiofacial syndrome. Neuroscience 164:257-271.

Wechsler D (1997) The Wechsler adult intelligence scale-III: administration and scoring manual. San Antonio, TX: The Psychological Corporation.

Wilson HR (1986) Responses of spatial mechanisms can explain positional hyperacuity. Vision Res 26:453-469.

Zangenehpour S, Cornish KM, Chaudhuri A (2009) Whole-brain expression analysis of FMRP in adult monkey and its relationship to cognitive deficits in fragile X syndrome. Brain Res 1264:76-84. 